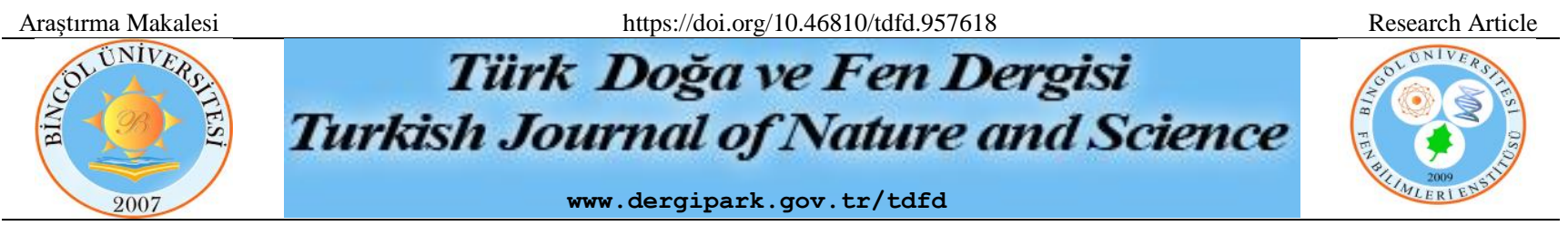

\title{
Meme Kanseri Tümörlerinin Derin Öğrenme Algoritmaları ile Sınıflandırılması
}

\author{
Seda Nur ÖZGÜR ${ }^{1}$, Sinem BOZKURT KESER ${ }^{1 *}$ \\ ${ }^{1}$ Eskişehir Osmangazi Üniversitesi, Mühendislik-Mimarlık Fakültesi, Bilgisayar Mühendisliği Bölümü, Eskişehir, \\ Türkiye \\ Seda Nur ÖZGÜR ORCID No: 0000-0001-8771-234X \\ Sinem BOZKURT KESER ORCID No: 0000-0002-8013-6922 \\ *Sorumlu yazar:sbozkurt@ogu.edu.tr
}

(Alınış: 25.06.2021, Kabul: 17.10.2021, Online Yayınlanma: 31.12.2021)

\begin{abstract}
Anahtar
Kelimeler

Meme

Kanseri,

Mamogram,

Derin

Öğrenme,

Siniflandirma,

Inception-

ResNet-V2

Öz: Meme kanseri, kadınlarda ölümlere neden olabilen hastalıklar arasında en başlarda gelen hastalıklardan biridir. Yapılan araştırmalara göre meme kanserinin erken teşhisi ile ölüm oranları düşürülebilmektedir. Meme kanserinin teşhisinde incelenen mamogram görüntülerinin radyologlar tarafindan incelenmesi uzun zaman almakta hatta zaman zaman bu incelemelerde hatalı sonuçlar elde edilebilmektedir. Meme kanserinin erken aşamalarda teşhis edilebilmesi için yapay zekâ yöntemleri kullanılarak yapılan çalışmalar oldukça önemlidir. Gelişen teknolojiyle birlikte birçok farklı derin öğrenme modeli bu hastalığın teşhisinde kullanılmaktadır. Bu çalışmada, meme kanserinin teşhisi için Inception-ResNet-V2 derin öğrenme modeli önerilmektedir. Önerilen derin öğrenme modeli, Inception ve ResNet modellerinin melezi bir mimari olup etkili bir şekilde geliştirilmiş sınıflandırma ve tanıma performansına sahiptir. Önerilen derin öğrenme mimarisi sırasıyla önişleme, sınıflandırma ve performans değerlendirme olmak üzere üç aşamadan oluşmaktadır. Önişleme aşamasında gerçek veri setlerinden alınan mamogram görüntüleri dolgu ile maskelenerek kesin bölgeyi tanımlamak amacıyla ilgi bölgeleri çıkarılmıştır. Böylece mamografi görüntülerinden bölgesel yoğunluklar tespit edilerek kanserli bölgenin konumu saptanır. Bu işlem sınıflandırma işlemi kolaylaştırmak için yapılan bir önişlemdir. İlgi bölgelerinin tespitin ardından, segmentasyon ișlemi ile bilgi içermeyen bölümler kırpılır. Yeniden boyutlandırma son önişleminin ardından önerilen Inception-ResNet-V2 derin öğrenme modeli ile sınıflandırma işlemi gerçekleştirilmektedir. Önerilen model ile \%96.21 doğruluk, \%97.48 geri çağırma, \%98.18 kesinlik, \%97.83 F-ölçütü, \%98.00 eğri altında kalan alan ve 0.83 cohen kappa performans değerleri elde edilmiştir. Elde edilen sonuçlar, çalışmada kıyaslama aşamasında kullanılan diğer derin öğrenme mimarilerinden elde edilen sonuçlar ile karşılaştırıldığında önerilen modelin meme kanseri teşhisinde daha iyi performans sergilediğini kanıtlamaktadır.
\end{abstract}

\section{Classification of Breast Cancer Tumors with Deep Learning Algorithms}

Keywords
Breast Cancer,
Mammogram,
Deep
Learning,
Classification,
Inception-
ResNet-V2

Keywords Mammogram, Deep Learning, InceptionResNet-V2

\begin{abstract}
Breast cancer is one of the leading diseases among diseases that can cause death in women. Studies have shown that early detection of breast cancer can reduce mortality rates. The mammogram images used in the diagnosis of breast cancer are examined by radiologists. Investigations take a long time and sometimes erroneous results can be obtained. Studies using artificial intelligence methods are very important for the diagnosis of breast cancer in the early stages. It has also been observed that the diagnoses obtained with mammogram images are better than the diagnosis of medical experts. With the developing technology, many different deep learning models are used in the diagnosis of the disease. In this study, the Inception-ResNet-V2 deep learning model is proposed for the diagnosis of breast cancer. The proposed deep learning model is a hybrid architecture of Inception and ResNet models and has effectively improved classification and diagnosis performance. The proposed deep learning architecture consists of three stages, namely preprocessing, classification, and performance evaluation, respectively. In the preprocessing stage, mammogram images from real datasets were masked with filler, and regions of interest were extracted to define the precise region. Thus, regional densities are detected from mammography images and the location of the cancerous area is determined. This is a pre-process
\end{abstract}


to simplify the classification process. After detection of regions of interest, segments that do not contain information are clipped by segmentation. After the resizing post-processing, the classification process is carried out with the proposed Inception-ResNet-V2 deep learning model. With the proposed model, $96.21 \%$ accuracy, $97.48 \%$ recall, $98.18 \%$ precision, $97.83 \%$ F-score, $98.00 \%$ area under the curve, and 0.83 cohen kappa performance values were obtained. The obtained results prove that the proposed model performs better in breast cancer diagnosis when compared with the results obtained from other deep learning architectures used in the benchmarking stage in the study.

\section{GíRíş}

Organ veya dokudaki hücrelerin düzensiz olarak bölünerek çoğalması sonucu oluşan kötü huylu tümörler kanser olarak adlandırılmaktadır. Kanser tipleri, anormal hücrelerin kontrolsüz şekilde çoğalması ile başlamaktadır [1]. Meme kanseri dünya çapında en yaygın sağlık problemi olmaya devam etmekte olan kanser türleri arasındadır. Meme kanseri kadınlarda yaşamı tehdit eden bir hastalıktır ve kadın nüfusu arasında ölümlerin önde gelen sebeplerinden biridir [2].

2018 yılında, dünya çapında 2,08 milyon yeni meme kanseri vakası ve meme kanserinden dolayı 627.000 ölüm gerçekleşmiştir [3]. Erkeklerde meme kanseri görülme olasılığı kadınların \%1'i kadardır. İnsani Gelişme Göstergesi (Human Development Index) düşük veya orta olan ülkelerde meme kanseri ölüm oran1 \%48'dir. Bu oran İnsani Gelişme Göstergesi yüksek veya çok yüksek olan ülkelerdekinin 4 katıdır. Etkili tarama programları ile meme kanserinin erken teşhisi, meme kanseri tedavi sürecini olumlu etkilemektedir. Mamografi taraması ile yüksek gelirli ülkelerde meme kanserinden ölüm oranlarını önemli ölçüde azalmıştır [4]. Dünyada, meme kanseri riski altındaki sağlam kişilerin belirli sürede, belirli bir hastalığa yakalanma olasılığı (insidans oranı) ve bu hastalıktan ölen kişilerin oranları sırasıyla Şekil 1 (a) ve (b) ile gösterilmektedir.
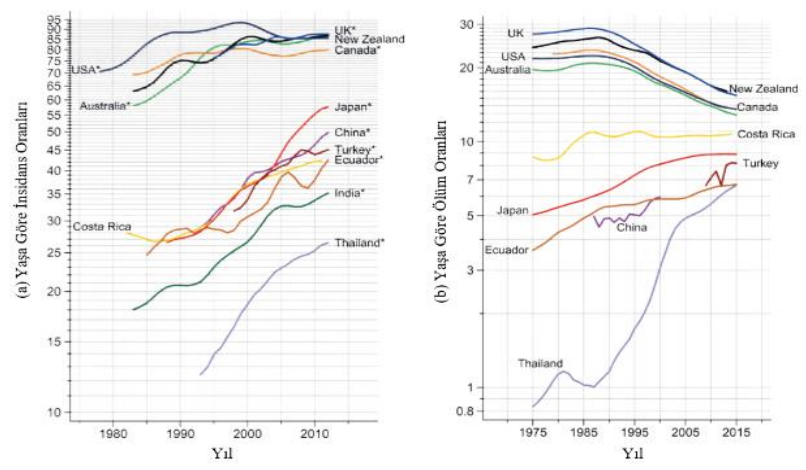

Şekil 1. Dünyada yaşa göre standartlaştırılmış (a) insidans oranları ve (b) ölüm oranları

Belirli bir ülkede belirli bir sürede tespit edilen yeni meme kanseri vaka sayısının o ülkedeki yıl ortasındaki nüfusa veya risk altındaki kişi sayısına bölünmesi ile insidans oranı elde edilir. Şekil 1 incelendiğinde, Türkiye'de insidans ve ölüm oranlarında artış olduğu gözlenmektedir. Erken teşhis ve etkili tarama programları aracılığıyla meme kanserinin daha erken saptanması sayesinde USA, UK vb. ülkeler gibi Türkiye'de de bu oranlarda düşüş olması beklenmektedir. Araştırma sonuçlarına göre meme kanserinin erken teşhisi ile ölüm oranları önemli ölçüde düşürülebilmektedir. Meme kanserinin teşhisinde, radyologlar tarafindan incelen mamogram görüntüleri ile hastalığın gidişatı hakkında bilgi edinmek mümkündür. Şekil 2'de iyi huylu (benign) ve kötü huylu (malignant) meme dokularına ait mamogram görüntüleri verilmektedir.

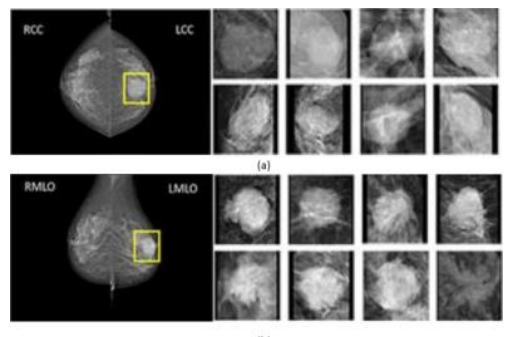

Şekil 2. (a) İyi huylu ve (b) kötü huylu meme kanseri mamogramı

Yapay zeka teknolojilerin gelişmesi ile birlikte hastalıkların teşhisinde önemli gelişmeler yaşanmaktadır. Son zamanlarda, genel olarak derin öğrenme tekniklerinin ve evrişimli sinir ağlarının tanıtılmasıyla yapay zeka alanında bir atılım gerçekleştirilmiştir. Bu tür teknikler, bir özellik alanı tanımlamasına ihtiyaç duymaz ve insan uzmanları bile geride birakabilecek siniflandirma performansları elde edebilirler [5]. Mamograma dayalı meme kanseri risk değerlendirmesi için derin öğrenme yaklaşımıyla elde edilen sonuçlar umut vericidir. Derin öğrenme, ham görüntü verilerinden birden çok gizli katmana sahip sinir ağları aracılığıyla denetimli (supervised) bir modda veya otomatik kodlayıcılar (autoencoders) aracılığıyla denetimsiz (unsupervised) bir modda, örüntü algılama ve sınıflandırma için yararlı olan hiyerarşik temsillerin otomatik öğrenmesini içerir. Ayrıca, büyük verinin ve makine öğreniminin kullanılması ile özellikle görüntütabanlı tarama için tarama testlerinin doğruluğunu iyileştirmek ve tarama protokollerine daha iyi rehberlik etmek için yeni firsatlar sunulmaktadır [6].

Bu çalışmada, meme kanseri tümörlerinin tespit edilmesi amaçlanmaktadır. Meme kanserinin tespiti için Inception-ResNet-V2 sinıflandırma modeli önerilmiştir. Gerçek veri seti kullanılarak, önerilen yöntemin performansı değerlendirilmiştir. Önerilen derin öğrenme modeli, Inception ve ResNet modellerinin melezi bir mimari olup etkili bir şekilde geliştirilmiş sınıflandırma ve tanıma performansına sahiptir. Önerilen derin öğrenme mimarisi sırasıyla önişleme, sınıflandırma ve performans değerlendirme olmak üzere üç aşamadan oluşmaktadır. Önişleme aşamasında gerçek veri setlerinden alınan mamogram görüntüleri dolgu ile maskelenerek kesin bölgeyi tanımlamak amacıyla ilgi 
bölgeleri çıkarılmıştır. Böylece mamografi görüntülerinden bölgesel yoğunluklar tespit edilerek kanserli bölgenin konumu saptanır. $\mathrm{Bu}$ işlem sınıflandırma işlemi kolaylaştırmak için yapılan bir önişlemdir. İlgi bölgelerinin tespitin ardından, segmentasyon işlemi ile bilgi içermeyen bölümler kırpılır. Yeniden boyutlandırma son önişleminin ardından önerilen Inception-ResNet-V2 derin öğrenme modeli ile sınıflandırma işlemi gerçekleştirilmektedir. Gerçek veri seti kullanılarak, önerilen yöntemin performansı değerlendirilmiştir. Deneylerde \%96.21 doğruluk, \%97.48 geri çağırma, \%98.18 kesinlik, \%97.83 F-ölçütü, \%98.00 eğri altında kalan alan ve 0.83 cohen kappa değerleri elde edilmiştir. Çalışmada kullanılan diğer derin öğrenme mimarileri ile karşılaştırıldığında önerilen yöntemin meme kanseri teşhisinde daha başarılı olduğunu kanıtlanmaktadır. Makalenin takip eden bölümünde literatürde yapılan çalışmalar, kullanılan veri seti, önerilen yöntem ve performans değerlendirme ölçütleri verilmektedir. Üçüncü bölümde deney sonuçları yer almaktadır. Makale, sonuçlar ve gelecekte yapılacak çalışmalar ile sonlandirılmaktadir.

\section{MATERYAL VE METOT}

\subsection{Literatürde Yapılan Çalışmalar}

Meme kanseri için erken teşhis oldukça önemlidir ve ölüm oranını etkili bir şekilde azaltmaktadır. Bu sebeple son yıllarda derin öğrenme ve makine öğrenmesi algoritmaları kullanılarak yapılan meme kanseri teşhis çalışmalarına ilgi artmaktadır. Mamogram görüntülerinin, derin öğrenme ve makine öğrenme algoritmaları ile kullanılmasıyla erken teşhiste başarılı sonuçlar elde edilmektedir.

Literatürde meme kanserini erken teşhis etmek için çeşitli çalışmalar yapılmaktadır. Jadoon vd. [7] 2017 yılında Mamografi için Dijital Veri Tabanı (DDSM, Digital Database for Screening Mammography) üzerinde Destek Vektör Makinesi (SVM, Support Vector Machine) ile Evrişimli Sinir Ağı (CNN, Convolutional Neural Network) algoritmalarını uygulamışlardır. Deneylerde, CNN algoritmasının \%83.74 doğruluk oranı (ACC, Accuracy) ile daha başarılı olduğu sonucuna ulaşılmıştır. Hepsağ, Özel ve Yazıcı [8] (2017) yaptıkları çalışmada, Mamografik Görüntü Analizi Topluluğu (Mini-MIAS, Mammographic Image Analysıs Society) ve Meme Kanseri Dijital Depo (BCDR, Breast Cancer Digital Repository) üzerinden elde edilen veri setleri üzerinde $\mathrm{CNN}$ derin öğrenme modelini kullanarak meme kanserini iyi huylu ve kötü huylu olarak sınıflandırmışlardır. Bu çalışmada, en yüksek başarı Mini-MIAS veri seti ile \%68 ACC, \%59 Kesinlik (PRE, Precision), \%55 Geri Çağırma (Recall) ve \%57 F-Ölçütü (F-Score) olarak bulunmuştur. Nahid vd. [9] 2018'de yaptığı çalışmada, meme kanseri görüntülerinin sınıflandırılması için CNN ve Uzun-Kısa Süreli Bellek (LSTM, Long Short Term Memory) yöntemlerinin bir arada kullanımını önermektedirler. Softmax ve SVM katmanları, önerilen yeni Derin Sinir Ağ 1 (DNN, Deep Neural Network) modelleri ile özellikler çıkarıldıktan sonra karar verme aşamasında kullanmıștır. Deney sonuçlarında, CNN ve Softmax birlikte kullanılarak \%91 ACC, \%93 Duyarlılık (SEN, Sensitivity) ve \%96 PRE ile en iyi değerler elde edilmiştir. Zhang vd. [10] (2018) yürüttükleri çalışmada Mini-MIAS veri setini kullanmışlardır. Veri arttırma tekniği ile veri setinin boyutunu arttırılarak dokuz katmanlı CNN mimarisini önerilmiştir. $\mathrm{Bu}$ çalışmada, düzeltilmiş doğrusal Doğrultulmuş Doğrusal Birim (ReLU, Rectified Linear Unit), sızdıran ReLU (leaky ReLU) ve parametrik ReLU (parametric ReLU) olmak üzere üç farklı aktivasyon fonksiyonu karşılaştırılmaktadır. \%94 ACC, \%93.4 SEN, \%96 PRE ile en iyi performans değerleri elde edilmiştir. Sivasakthiselvan vd. [11] 2018 y1lında yaptıkları çalışmada, görüntülerden özellik çıkarma işlemi ve İlgi Bölgesi (ROI, Region of Interest) segmentasyonu yapıldiktan sonra 2-boyutlu CNN ve 3-boyutlu CNN mimarileri sınıflandırma aşamasında kullanılımı ile \%71 ACC, \%81 SEN ve \%76 AUC performans değerlerini elde etmişlerdir. Behera vd. [12] (2018), DenseNet-II sinir ağ1 modelini önermişlerdir. Deneylerde AlexNet, VGGNet, GoogLeNet ve DenseNet modellerini karşılaştırma aşamasında kullanmışlardır. Deney sonuçlarında, \%94.55 ACC ve \%95.6 SEN değerleri ile önerilen yöntemin diğer modellerden üstünlüğü gösterilmiştir. Ahmed vd. [13] (2018), INbreast veri seti üzerinde CNN mimarisini uygulamışlardır. Deney sonuçlarında, \%80.1 ACC, \%80 SEN ve \%78 Eğri Altında Kalan (AUC, Area Under the Curve) değerleri elde edilmiştir. Ragab vd. [14] (2019), derin öğrenme ve bazı segmentasyon tekniklerini kullanarak meme kanserini iyi huylu ve kötü huylu olarak sınıflandırmışlardır. ROI manuel kırpma yöntemi ile $\% 79$ ACC, \%76.3 SEN, \%85 PRE ve \%88 AUC değerleri elde edilmiştir. Eşik ve bölge-bazlı segmentasyon tekniği ile \%80.5 ACC, \%77.4 SEN, \%86 PRE ve \%88 AUC değerleri elde edilmiştir. Duggento vd. [5] (2019), mamogram görüntülerinden meme lezyonu sınıflandırması için $\mathrm{CNN}$ mimarisini kullanmışlardır. Çalışmada kabul edilebilir doğruluk değerleri korunurken yanlış negatiflerin azaltılması hedeflenmiştir. DDSM üzerinden elde edilen Küratörlü Göğüs Görüntüleme Alt Kümesi (CBIS-DDSM, Curated Breast Imaging Subset of DDSM) veri seti ile gerçekleştirilen deneylerde \%71 ACC, \%84.4 SEN ve $\% 77$ AUC sonuçlarına ulaşılmıştır. Li vd. [15] (2019) VGGNet, GoogleNet, DenseNet ve DenseNet-II sinir ağ 1 modellerini FFDM veri seti üzerinde, meme kanseri verilerinin iyi huylu ve kötü huylu olarak sınıflandırılması üzerinde çalışmışlardır. Deneylerde, en iyi sonuçlar DenseNet-II mimarisi ile elde edilmiştir. Chanda ve Sarkar [16] (2019), mamogram görüntülerini segmentasyon aşamasından geçirdikten sonra bölgeyi tanımlamaya çalışmışlardır. Bölümlere ayrılmış mamogram görüntülerinden yoğunluk, doku ve şekiller gibi özellikler çıkarılmıştır. Segmentasyon işlemi ile verilerin hızlı şekilde sinıflandırılabildiğini kanıtlamışlardır. Deneylerde, \%89 ACC, \%74 Özgüllük (Spe, Specificity) ve \%89 SEN sonuçlarına ulaşılmıştır. Trivizakis vd. [17] (2019) mamogram görüntülerinden meme yoğunluğu skoru tespiti için Transfer Öğrenimi (Transfer Learning) ve ImageNet algoritmalarını önermişlerdir. Önerilen yöntemler, DDSM ve Mini- 
MIAS veri setleri kullanılarak analiz edilmiştir. Deney sonuçlarında, DDSM veri seti ile \%79.3 ACC ve \%78 AUC sonuçlarına ulaşılmıştır. Yu vd. [18] (2020), MiniMIAS veri setini kullanarak önceden eğitilmiş modellere dayalı derin füzyon öğrenme yaklaşımını uygulamışlardır. Deneylerde, \%89 ACC, \%91 geri çağırma ve \%90 PRE sonuçları elde edilmiştir.

Literatürde meme kanseri teşhisinde derin öğrenme modelleri ile gerçekleştirilen çalışmalar Tablo 1'de özetlenmektedir.

Tablo 1. Derin Öğrenme Modelleri ile Meme Kanseri Teşhisinde Literatürde Yapılan Çalışmalar

\begin{tabular}{|c|c|c|c|c|c|c|}
\hline Yazar & Yöntem & Veri Seti & $\mathrm{ACC}$ & SEN & PRE & AUC \\
\hline $\begin{array}{l}\text { Jadoon vd. } \\
\text { (Ocak 2017) }\end{array}$ & $\begin{array}{c}\text { CNN + wavelet } \\
\text { CNN + SVM }\end{array}$ & DDSM & $\begin{array}{l}\% 81.83 \\
\% 83.74\end{array}$ & - & - & $\begin{array}{l}\% 83.10 \\
\% 83.90\end{array}$ \\
\hline $\begin{array}{l}\text { Hepsağ, Özel ve Yazıcı } \\
\text { (Ekim 2017) }\end{array}$ & $\mathrm{CNN}$ & Mini-MIAS & $\% 68.00$ & - & $\% 59.00$ & - \\
\hline $\begin{array}{l}\text { Nahid vd. } \\
\text { (Mart 2018) }\end{array}$ & CNN+Softmax & BreakHis & $\% 91.00$ & $\% 93.00$ & $\% 96.00$ & - \\
\hline $\begin{array}{c}\text { Zhang vd. } \\
\text { (Temmuz 2018) }\end{array}$ & CNN-PReLU-RSP & Mini-MIAS & $\% 94.00$ & $\% 93.40$ & $\% 94.50$ & - \\
\hline $\begin{array}{l}\text { Sivasakthiselvan vd. } \\
\text { (Ağustos 2018) }\end{array}$ & 3DCNN & 143 Hastanın görsel verisi & $\% 71.00$ & $\% 81.00$ & - & $\% 76.00$ \\
\hline $\begin{array}{c}\text { Behera vd. } \\
\text { (Eylül 2018) }\end{array}$ & DenseNet-II & FFDM & $\% 94.55$ & $\% 95.60$ & - & - \\
\hline $\begin{array}{l}\text { Ahmed vd. } \\
\text { (Aralık 2018) }\end{array}$ & CNN & INbreast & $\% 80.10$ & $\% 80.00$ & - & $\% 76.00$ \\
\hline $\begin{array}{l}\text { Ragab vd. } \\
\text { (Ocak 2019) }\end{array}$ & $\begin{array}{c}\text { CNN } \\
\text { ROI threshold }\end{array}$ & $\begin{array}{c}\text { DDSM } \\
\text { CBIS-DDSM }\end{array}$ & $\begin{array}{l}\% 79.00 \\
\% 80.50\end{array}$ & $\begin{array}{l}\% 76.30 \\
\% 77.40\end{array}$ & $\begin{array}{l}\% 82.20 \\
\% 86.00\end{array}$ & $\begin{array}{l}\% 88.00 \\
\% 88.00\end{array}$ \\
\hline $\begin{array}{l}\text { Duggento vd. } \\
\text { (Mayıs 2019) }\end{array}$ & $\mathrm{CNN}$ & CBIS-DDSM & $\% 71.00$ & $\% 84.40$ & - & $\% 77.00$ \\
\hline $\begin{array}{c}\text { Li vd. } \\
\text { (Mayis 2019) } \\
\end{array}$ & DenseNet-II & FFDM & $\% 94.55$ & $\% 95.60$ & - & - \\
\hline $\begin{array}{c}\text { Chanda ve Sarkar } \\
\text { (Eylül 2020) }\end{array}$ & Efficient Image Segmentation Technique & - & $\% 89.00$ & $\% 89.00$ & - & - \\
\hline $\begin{array}{l}\text { Trivizakis vd. } \\
\text { (Kasım 2019) }\end{array}$ & $\begin{array}{c}\text { Transfer learning } \\
\text { ImageNet }\end{array}$ & $\begin{array}{c}\text { DDSM } \\
\text { Mini-MIAS }\end{array}$ & $\begin{array}{l}\% 74.80 \\
\% 79.30 \\
\end{array}$ & - & - & $\begin{array}{l}\% 84.20 \\
\% 78.00 \\
\end{array}$ \\
\hline $\begin{array}{c}\text { Yu vd. } \\
\text { (Eylül 2020) }\end{array}$ & VGG19_Fusion1 & Mini-MIAS & $\% 89.00$ & - & $\% 90.00$ & - \\
\hline $\begin{array}{c}\text { Roy vd. } \\
\text { (Eylül 2021) }\end{array}$ & $\begin{array}{l}\text { CNN, CCA, K-means, } \\
\text { Fuzzy c-means }\end{array}$ & DDSM & $\% 90$ & - & - & - \\
\hline George (2021) & M-ELBP & DDSM & $\% 86.47$ & - & - & - \\
\hline
\end{tabular}

\subsection{Veri Seti}

Meme kanserinin tespitinde kullanılan mamogram görüntüleri meme kanserinin erken teşhisi için büyük bir öneme sahiptir. Son y1llarda derin öğrenme ve makine öğrenmesi yöntemleri ile mamogram görüntüleri kullanılarak sınıflandırma çalışmaları yapılmaktadır. Bu çalışmada, DDSM ve CBIS-DDSM veri setlerinden alınan mamogram görüntüleri kullanılmıştır (Şekil 3). Veri setinde sağlıklı, iyi huylu kireçlenme, iyi huylu kitle, kötü huylu kireçlenme ve kötü huylu kitleye sahip 55885 adet veri bulunmaktadır. Ayrıca her iki göğüs içinde hem CC (Kraniokaudal) hem MLO (Mediolateral oblik) görünümlerini mevcuttur. Veri setindeki görüntülere ROI segmentasyonu yapılarak görüntüler 299x299 olarak yeniden boyutlandırılmıştır.

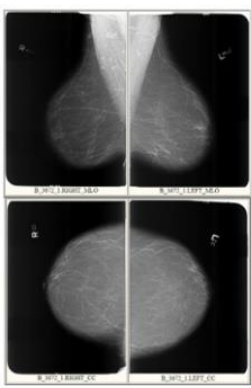

(a)
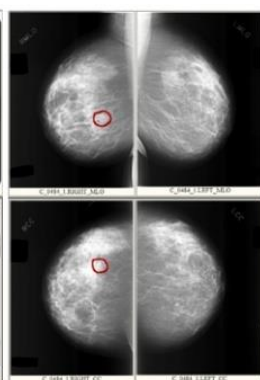

(b)

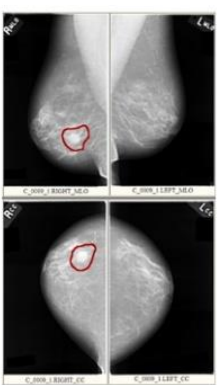

(c)
Şekil 3. (a) Sağlıklı (b) İyi Huylu (c) Kötü Huylu Mamogram Görüntüleri

\section{3. Önerilen Yöntem}

Kadınlarda ölüme sebep olan hastalıklar arasında ilk siralarda meme kanseri gelmektedir. Meme kanserinin ilk aşamalarda tespit edilebilmesiyle ölüm oranları büyük ölçüde düşürülebilmektedir. Son zamanlarda yapay zekâ teknikleri kullanılarak yapılan çalışmalar erken teşhis için umut vadetmektedir. $\mathrm{Bu}$ çalışmada, mamogram görüntülerin yapay zekâ teknikleri kullanılarak tespit edilmesi önerilmektedir. Önerilen derin öğrenme modelinde, ilk olarak DDSM veri setinde bulunan mamogram görüntüleri ön işleme aşamasından geçirilmiştir. Daha sonra Inception-Resnet-V2 mimarisi ile meme kanseri iyi huylu ve kötü huylu olarak sınıflandırılmıştır. Şekil 4'te önerilen derin öğrenme modeline ait akış diyagramı verilmektedir. Şekil 4 ile verilen akış diyagramına ait adımlar alt bölümlerde detaylandırılmaktadır. 


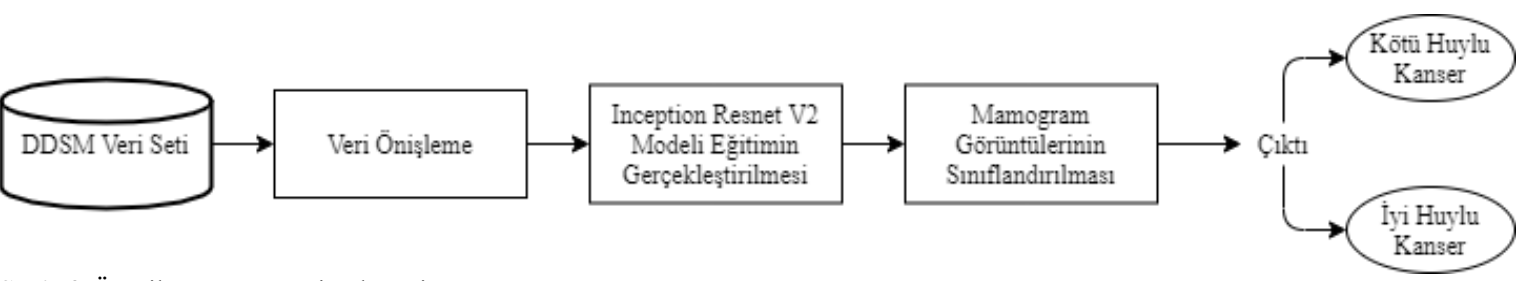

Şekil 4. Önerilen Yönteme ait Akış Diyagram

\subsubsection{Veri önişleme}

Çalışmada kullanılan DDSM ve CBIS-DDSM veri setlerinden alınan mamogram görüntüleri dolgu (padding) ile maskelenerek (masking) ilgi bölgeleri (ROI, Region of Interest) çıkarılmıştır. ROI mamogram görüntülerinden kesin bölgeyi tanımlamak için kullanılan önemli bir işlemdir. $\mathrm{Bu}$ işlem ultrason veya mamografi gibi görüntülerden bölgesel yoğunlukları tespit ederek kanserli bölgenin konumunu belirlemek ve sınıflandırmayı kolaylaştırmak için yapılmaktadır. Görüntülerden bilgi içermeyen bölümlerin çıkarılması işlemidir. İlgi bölgeleri tespit edilen görüntülerden diğer bölgelerin kırpılmasına segmentasyon adı verilmektedir. $\mathrm{Bu}$ çalışmada ROI segmentasyonu yapılan görüntüler daha sonra rastgele olarak döndürülmektedir. Elde edilen mamogram görüntüleri önce $299 \times 299$ olarak yeniden şekillendirilmiştir. Daha sonra $100 \times 100$ olarak yeniden

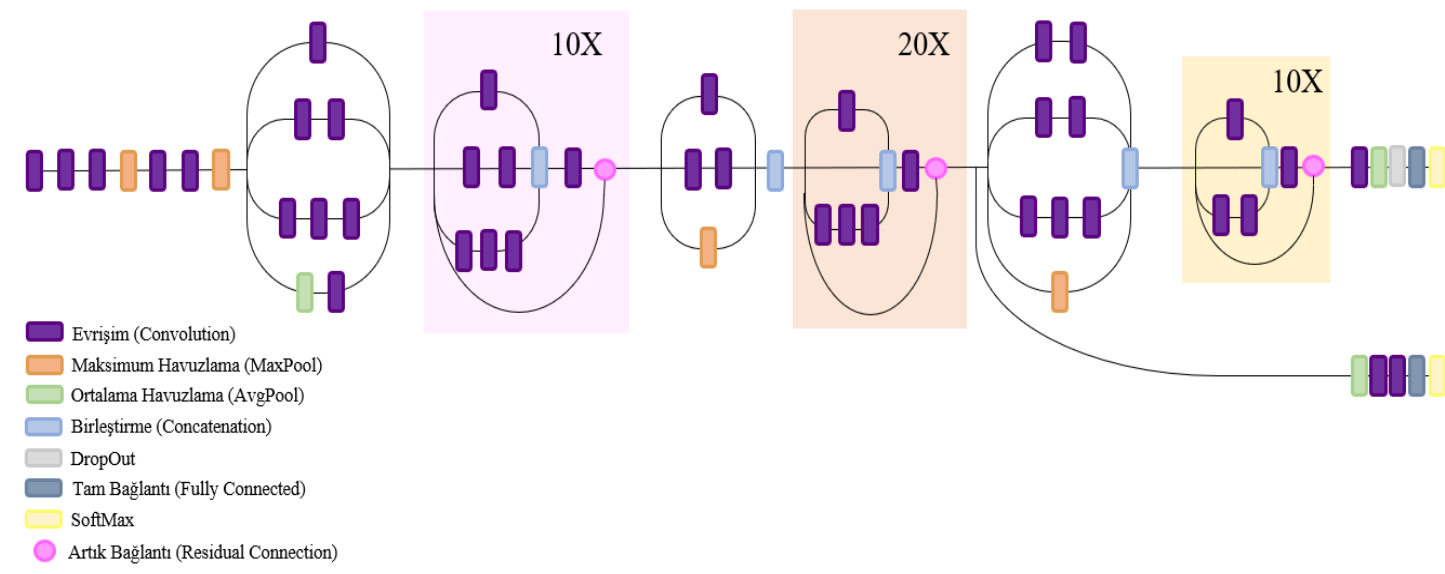

boyutlandırılmıştır. Son olarak, veri setinde bulunan görüntüler $\% 80$ eğitim, $\% 20$ test olmak üzere ayrılmaktadır. Şekil 5 (a) ve (b) ile sırasıyla önişleme öncesi ve sonrası elde edilen mamogram görüntüleri verilmektedir.

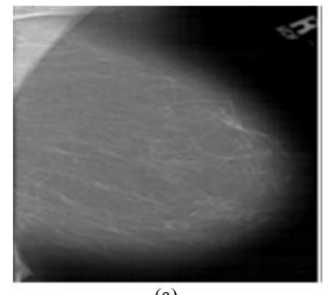

(a)

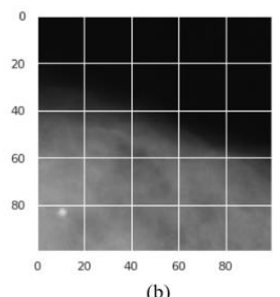

(b)

Şekil 5. (a) Önişleme Öncesi Mamogram Görüntüsü (b) Önişleme Sonrası Mamogram Görüntüsü

Şekil 6. Inception-ResNet-V2 Ağ Mimarisi

\subsubsection{Inception-ResNet-V2 modeli}

$\mathrm{Bu}$ çalışmada Inception-ResNet-V2 mimarisi ile mamogram görüntülerinin sinıflandırılması önerilmektedir. Inception-ResNet-V2, geliştirilmiş tanıma ve sinıflandırma performansı ile Inception ve ResNet mimarilerinin bir araya getirilmesi ile oluşturulmuş bir modeldir. Resnet mimarisi derine, Inception ise enine gitmekle ilgilidir. $\mathrm{Bu}$ nedenle Inception-ResNet-V2 mimarisi ile hem derine hem enine gitmek konusunda optimum sonucu elde edebiliriz. Inception-ResNet-V2, Inception mimari ailesini temel alan ve artık bağlantıları (residual connection) içeren evrişimli sinir ağı algoritmasıdır (Şekil 6). Artık bağlantılar, model eğitilirken kısa yollara izin vererek araştırmacıların, daha iyi performansa ulaşmalarını sağlayan daha derin sinir ağlarını başarılı bir şekilde eğitmelerini sağlamaktadır. Bu aynı zamanda başlangıç bloklarının önemli ölçüde basitleştirilmesini de sağlar. $\mathrm{Bu}$ yap1, ilk evrişim işleminin boyutunu $1 \times 1$ olarak değiștirerek artık katmanın optimizasyonunu sağlar (Şekil 7). Ayrıca öğrenmenin durduğu koşullarda bile çıkışa bir önceki aktivasyon değerini aktarma üzerine kurulmuştur [19].

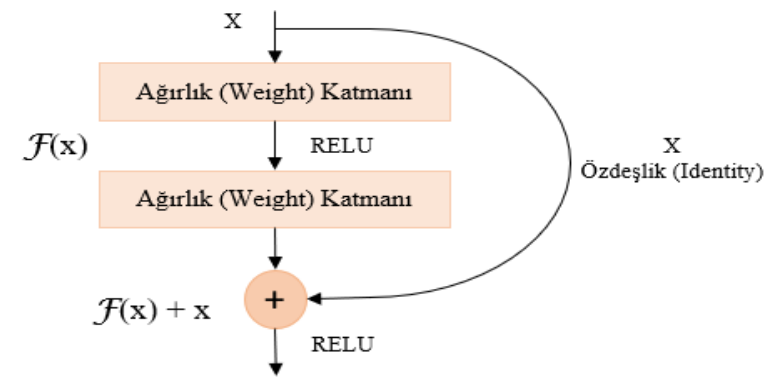

Şekil 7. Artık Bağlantı (Residual Connection 


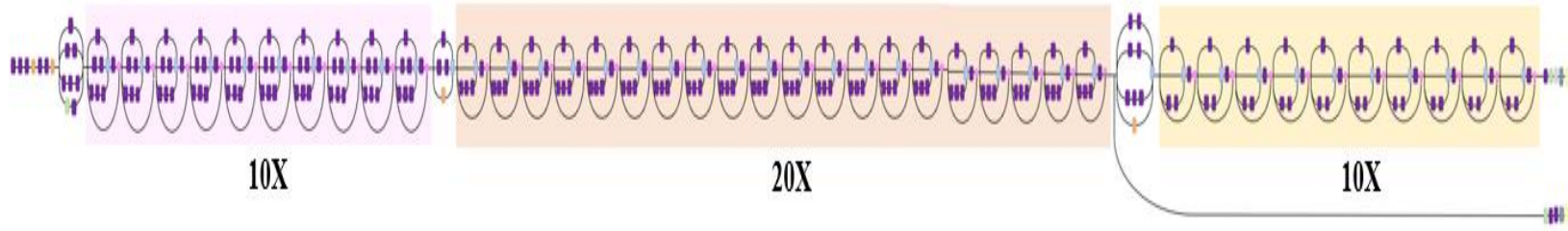

Şekil 8. Inception-ResNet-V2 Mimarisi (Sıkıştırılmış Görünümü)

\subsubsection{Performans değerlendirme ölçütleri}

$\mathrm{Bu}$ çalışmada, önerilen Inception-ResNet-V2 derin öğrenme modelinin etkinliği DDSM ve CBIS-DDSM veri setlerinden alınan mamogram görüntüleri kullanılarak analiz edilmiştir. Algoritmanın, meme kanseri teşhisinde üstünlüğünü kanıtlamak için diğer derin öğrenme modelleri ile karşılaştırmalar yapılmıştır. Karşılaştırma ölçütleri olarak; karmaşıklı matrisi (confusion matrix), doğruluk (accuracy), kesinlik (precision), geri çağırma (recall), F-ölçütü (F score), eğri altında kalan alan (area under the curve), Cohen kappa ölçütü (Cohen kappa score) performans değerlendirme ölçütleri kullanılmıştır.

Karmaşıklık Matrisi: Sınıflandırma işlemlerinde kullanılan bir performans ölçütüdür. Sınıf sayısı kadar sütun ve satırdan oluşan ve her hücrede farklı bir değer tutan tablodur (Tablo 2). Tahmin edilen ve gerçek değerlerden oluşan sayıları temsil etmektedir. Karmaşıklık matrisinden yararlanılarak doğruluk, kesinlik, geri çağırma, F-ölçütü gibi değerler hesaplanabilmektedir.

Tablo 2. Karmaşıklık Matrisi

\begin{tabular}{|c|c|c|c|}
\hline \multirow[b]{4}{*}{ 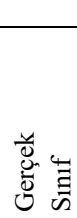 } & & \multicolumn{2}{|c|}{ Tahmin Edilen Sınıf } \\
\hline & & 0 & 1 \\
\hline & 0 & Doğru Negatif (DN) & Yanlış Negatif (YN) \\
\hline & 1 & Yanlış Pozitif(YP) & Doğru Pozitif (DP) \\
\hline
\end{tabular}

- Doğru Negatif: Hastanın iyi huylu kitleye sahip olduğunu ifade eder.

- Doğru Pozitif: Hastanın kötü huylu kitleye sahip olduğunu ifade eder

- Yanlış Negatif: Kötü huylu kitleye sahip olan kişiye yanlış bir şekilde iyi huylu kitle teşhisi koyulduğunu ifade eder.

- Yanlış Pozitif: İyi huylu kitleye sahip olan kişiye yanlış bir şekilde kötü huylu kitle teşhisi koyulduğunu ifade eder.

Doğruluk: Doğru olarak sınıflandırılan değerlerin tüm değerlere oranıdır.

$$
D \text { ğ } r u l u k=\frac{D N+D P}{D N+Y N+Y P+D P}
$$

Kesinlik: Doğru olarak sınıflandırılmış pozitif değerlerin tüm pozitif değerlere oranıdır.

$$
\text { Kesinlik }=\frac{D P}{Y P+D P}
$$

Geri Çağırma: Doğru olarak sınıflandırılmış pozitif değerlerin gerçek pozitif değerlere oranıdır.

$$
\text { Geri Çă̆ } ı r m a=\frac{D P}{Y N+D P}
$$

F-Ölçütü: Kesinlik ve geri çağırma değerlerinin harmonik ortalaması olarak hesaplanan performans ölçütüdür.

$$
\mathrm{F}-\text { Ölçütü }=\frac{2 * \text { Kesinlik } * \text { Geri Çă̆ } ı r m a}{\text { Kesinlik }+ \text { Geri Çağırma }}
$$

Eğri Altında Kalan Alan: Yöntemler arasında tutarlı bir karşılaştırma yapabilmek için kullanılan bir başka ölçüt ROC (Receiver Operator Characteristic) eğrisinin altında kalan alanın hesaplanmasıly elde edilmektedir. Positif sınıfi, negatif sınıftan ne kadar ayırabildiği sonucunu vermektedir. AUC değeri arttıkça sınıflar arasındaki ayrım yeteneği artmaktadır.

Cohen Kappa Ölçütü: Sınıflandırıcının ne kadar iyi bir performans gösterdiğinin sonucudur. Kategorik niteliklerin değerlendirilmesinde iki gözlemci arasındaki uyumu ölçen istatistiktir. Gözlemci sayısı ikiden çoksa Fleiss Kappa ölçütü kullanılmaktadır. Cohen kappa değeri +1 ile -1 arasında değerler almaktadır. Eğer Cohen kappa değeri +1 ise iki değerlendirme arasındaki uyumun tam olduğu söylenmektedir. Cohen kappa değeri 0 olursa şansa bağlı olarak uyumlu oldukları aksi halde aralarında uyum olmadığı söylenmektedir. Cohen kappa değeri -1 ise uyumsuzluğun tam olduğu söylenmektedir.

\section{DENEY SONUÇLARI}

Bu çalışmada, mamogram görüntüleri iyi huylu ve kötü huylu şeklinde sınıflandırılarak meme kanserinin erken teşhisine kaktı sağlanması hedeflenmektedir. Önerilen Inception-ResNet-V2 derin öğrenme modeli ile öncelikle mamogram görüntüleri dolgu ile maskelenerek ilgi bölgeleri çıkartılmıştır. Segmentasyon işlemi ile görüntüler kırpılmış ve kırpılan mamogram görüntüleri önce $299 \times 299$ boyutlarında yeniden şekillendirilmiştir. Daha sonra, $100 \times 100$ ölçümlerinde yeniden boyutlandırıldı. Sınıflandırma aşamasında InceptionResNet-V2 modeli ile elde edilen sonuçlar DenseNet121, DenseNet169, EfficientNet, InceptionV3, 
MobileNet, MobileNetV2, NasNetMobile, ResNet50, VGG16, VGG19 ve Xception derin öğrenme modelleri ile karşıllaştırılmıştır (Şekil 10). Deneyler Windows 10 işletim sisteminde Python 3.5.8 sürümü kullanılarak ve 12 GB RAM'e sahip (Intel® Core ${ }^{\mathrm{TM}}$ i5-7200U CPU @ $2.50 \mathrm{GHz} 2.71 \mathrm{GHz}$ ) bilgisayarda gerçekleştirilmiştir.
Deney sırasında kullanılan diğer derin öğrenme mimarileri Tablo 3 'te özetlenmiștir.

Tablo 3. Kullanılan Derin Öğrenme Mimarilerinin Açıklamaları

\begin{tabular}{|c|c|}
\hline Derin Öğrenme Mimarileri & Özellikleri \\
\hline DenseNet121 & $\begin{array}{l}\text { En önemli avantajı, özellik yayılımını gerçekleştirmek ve özelliğin yeniden kullanılmasına olanak } \\
\text { sağlayarak parametre sayısını indirgemektir. }\end{array}$ \\
\hline DenseNet169 & $\begin{array}{l}\text { DenseNet121 mimarisinin özelliklerine sahiptir. DenseNet169 mimarisi } 169 \text { katmandan oluşan } \\
\text { evrişimli sinir ağı mimarisidir. Görüntü sinıflandırma işlemlerinde sıklıkla tercih edilmektedir. }\end{array}$ \\
\hline EfficientNet & $\begin{array}{l}2019 \text { Senesinde Google araştırma ekibi tarafından önerilmiştir. Tasarımdaki temel ilke yapay sinir } \\
\text { ağının ölçeklenmesini orantısal şekilde yapmasıdır. }\end{array}$ \\
\hline InceptionV3 & Görüntü analizine ve nesne algılamaya yardımcı olmak için kullanılan evrișimli bir sinir ă̆ıdır. \\
\hline MobileNet & $\begin{array}{l}\text { Google Araştırma ekibinden Andrew G. Howard ve arkadaşları tarafindan önerilen derin öğrenme } \\
\text { modelidir. Sınıflandırma, algılama, yerleștirme ve segmentasyon gibi işlemlerde kullanılmaktadır. }\end{array}$ \\
\hline MobileNetV2 & MobileNet ile aynı özelliklere sahiptir, ama MobileNet modeline göre daha hızlı çalışmaktadır. \\
\hline NasNetMobile & $\begin{array}{l}\text { Pekiştirmeli ögrenme kullanılarak optimize edilmiş temel yapı taşlarından (hücrelerden) oluşan } \\
\text { ölçeklenebilir bir CNN mimarisidir. }\end{array}$ \\
\hline ResNet50 & 50 katman derinliğine ve artık bağlantılara sahip evrişimli sinir ağıdır. \\
\hline VGG16 & $\begin{array}{l}\text { Evrişim katmalarının 2'li ya da 3'lü kullanması özelliği ile diğer mimarilerden ayrılan basit bir ağ } \\
\text { modelidir. }\end{array}$ \\
\hline VGG19 & $\begin{array}{l}\text { Oxford Üniversitesi bünyesindeki Visual Geometry Group tarafindan önerilmiştir. VGG19 modeli } \\
\text { 143.667.240 parametreye sahiptir. }\end{array}$ \\
\hline Xception & $\begin{array}{l}\text { Derinlemesine ayrılabilir evrişimleri içeren derin bir evrişimli sinir ağı mimarisidir. Google } \\
\text { araştırmacıları tarafından geliştirilmiştir. }\end{array}$ \\
\hline
\end{tabular}



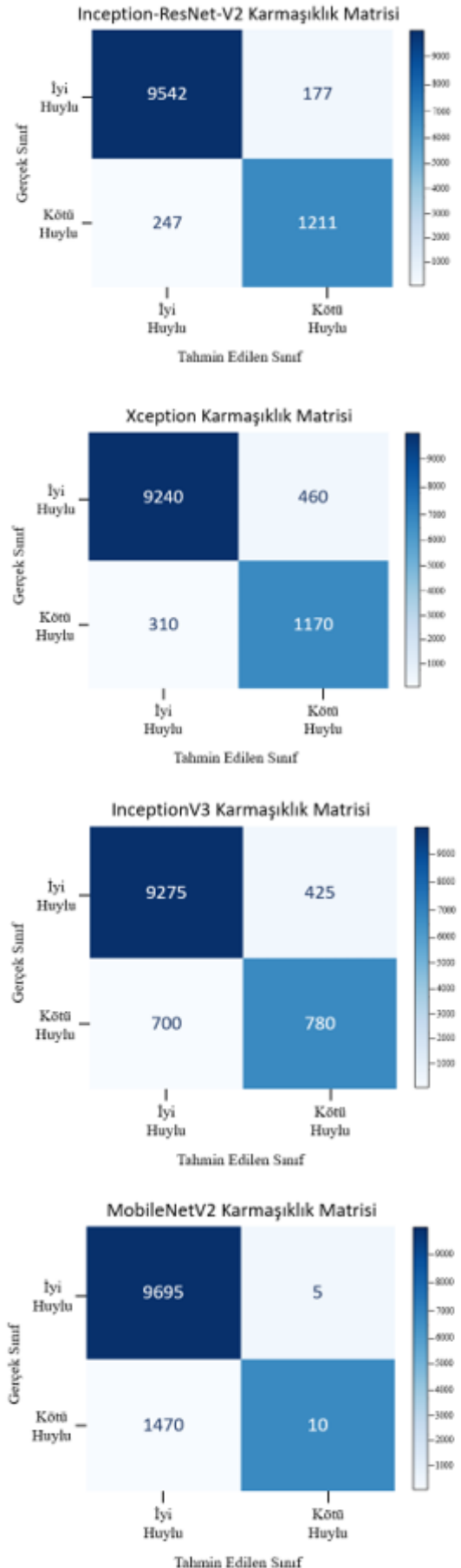

Şekil 9. Derin Öğrenme Modellerini2n Karmaşıklık Matrisleri Sonuçlar

Şekil 9 ile verilen karmaşıklık matrisleri ile tüm modellerin doğruluk, kayıp değeri (LOSS), geri çağırma (REC), kesinlik (PRE), F-ölçütü (FSC) ve AUC

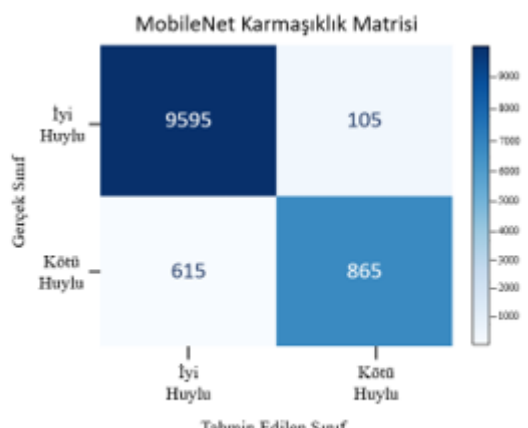

Tahmin Edilen Sinar
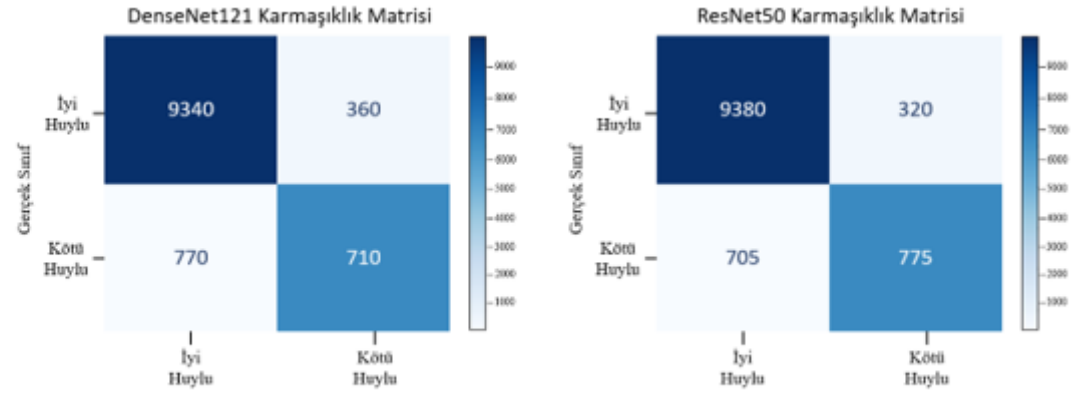

Tahmin Edilen Sunif
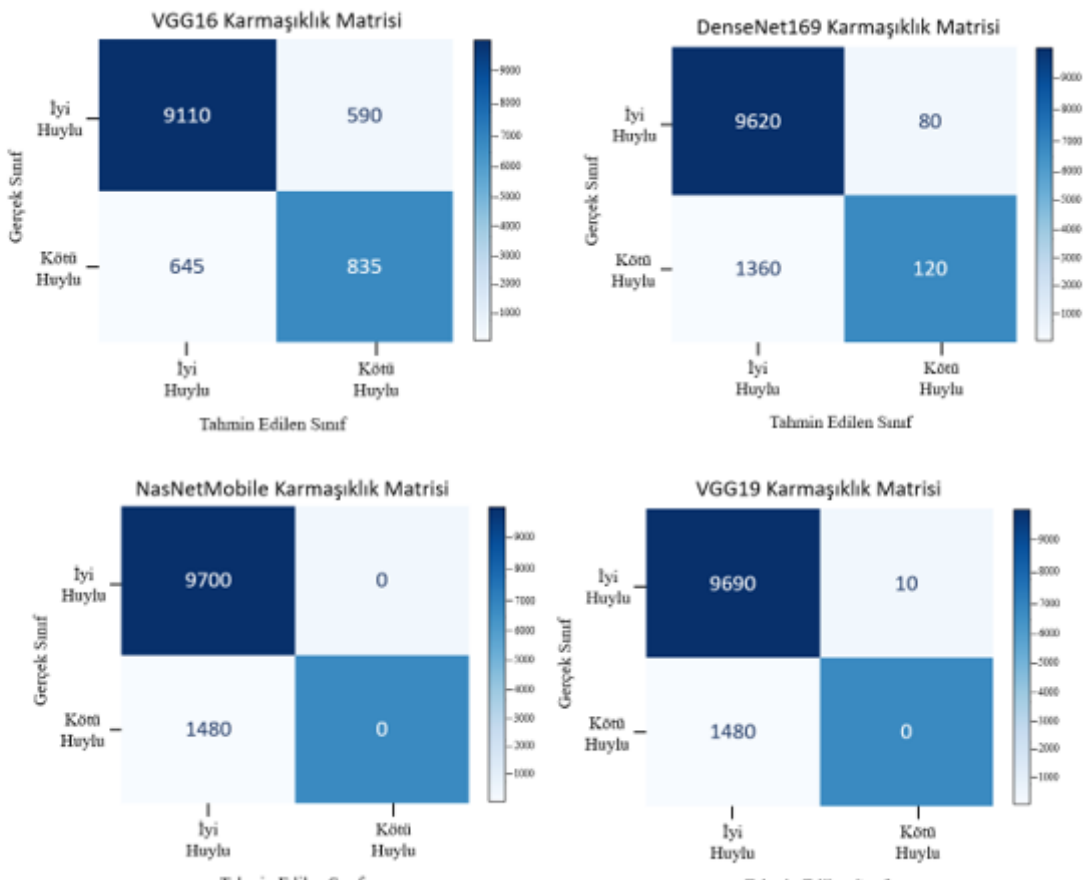

Tahmin Edilen Sanaf performans değerlendirme ölçütleri hesaplanmış ve Cohen Kappa (KAPPA) ölçütü ile önerilen modelin etkinliği ve performansı analiz edilmiştir (Tablo 4). 
Tablo 4. Deneylerde Kullanılan Derin Öğrenme Modellerin Performans Ölçütleri

\begin{tabular}{|c|c|c|c|c|c|c|c|}
\hline & ACC & LOSS & REC & PRE & FSC & KAPPA & AUC \\
\hline InceptionResNetV2 & $\mathbf{\% 9 6 . 2 1}$ & $\mathbf{\% 1 0 . 4 9}$ & $\mathbf{\% 9 7 . 4 8}$ & $\mathbf{\% 9 6 . 1 8}$ & $\mathbf{\% 9 7 . 8 3}$ & $\mathbf{0 . 8 3}$ & $\mathbf{\% 9 8 . 0 0}$ \\
\hline EfficientNet & $\% 94.14$ & $\% 15.64$ & $\% 97.78$ & $\% 95.41$ & $\% 96.58$ & 0.76 & $\% 97.00$ \\
\hline MobileNet & $\% 93.56$ & $\% 23.14$ & $\% 93.98$ & $\% 93.92$ & $\% 96.38$ & 0.67 & $\% 92.00$ \\
\hline Xception & $\% 93.11$ & $\% 18.85$ & $\% 96.75$ & $\% 93.26$ & $\% 96.00$ & 0.71 & $\% 95.00$ \\
\hline DenseNet121 & $\% 91.01$ & $\% 26.82$ & $\% 92.38$ & $\% 89.29$ & $\% 94.30$ & 0.50 & $\% 90.00$ \\
\hline ResNet50 & $\% 90.83$ & $\% 23.92$ & $\% 93.01$ & $\% 90.70$ & $\% 94.82$ & 0.55 & $\% 92.00$ \\
\hline InceptionV3 & $\% 90.00$ & $\% 25.68$ & $\% 92.98$ & $\% 89.62$ & $\% 94.28$ & 0.52 & $\% 90.00$ \\
\hline VGG16 & $\% 88.95$ & $\% 28.71$ & $\% 93.39$ & $\% 88.92$ & $\% 93.65$ & 0.51 & $\% 87.00$ \\
\hline DenseNet169 & $\% 87.12$ & $\% 32.88$ & $\% 87.61$ & $\% 84.18$ & $\% 93.04$ & 0.12 & $\% 79.00$ \\
\hline MobileNetV2 & $\% 86.81$ & $\% 35.79$ & $\% 86.83$ & $\% 87.95$ & $\% 92.93$ & 0.01 & $\% 80.00$ \\
\hline NASNetMobile & $\% 86.76$ & $\% 40.91$ & $\% 86.76$ & $\% 75.00$ & $\% 92.91$ & 0.00 & $\% 75.00$ \\
\hline VGG19 & $\% 86.67$ & $\% 39.44$ & $\% 86.75$ & $\% 75.00$ & $\% 92.86$ & -0.01 & $\% 85.00$ \\
\hline
\end{tabular}

Tablo 4 ile verilen sonuçlar incelendiğinde, diğer derin öğrenme modelleri ile kıyaslandığında önerilen Inception-ResNet-V2 derin öğrenme modeli ile en iyi değerler elde edilmiştir. Inception-ResNet-V2 modelinin evrişim katmanlarının hem derine hem de enine büyüyebilmesi ve sahip olduğu artık bağlantılar, diğer derin öğrenme modellerinden daha yüksek başarı sergilemesine destek sağlamıştır. Şekil 9 ile verilen karmaşıklık matrislerinde, önerilen model ile her iki sınıf için de en doğru tahmin sonuçlarının elde edildiği görülmektedir. Tıbbi teşhis araştırmalarında, PRE ve REC performansı raporlamak için kullanılan ana ölçütlerdir. FSC, tıbbi teşhislerde performansın raporlanmasında da önemlidir, çünkü kesinlik ve hatırlamayı karşılaştırmalar için kullanımı daha basit olan bir yöntem olan tek bir metrik altında birleştirir. Tıp camiasında, yanlış bir negatif normalde yanlış pozitiften daha yıkıcıdır. Yani, kötü huylu tümöre sahip hastaya,

Tablo 5. Derin Öğrenme Modellerin Gelenek Algoritmalar ile Birlikte Uygulanması Sonucu Elde Edilen Performans Ölçütleri iyi huylu etiketi verilmesi oldukça kritik bir hatadır. Bu nedenle PRE ve REC ölçümlerinin harmonik ortalaması olarak hesaplanan FSC daha iyi bir performans değerlendirme ölçütüdür. Tablo 4 ile verilen FSC değerleri incelendiğinde ise yine en iyi sonuçlara önerilen model ile erişildiğini söyleyebiliriz. Önerilen modelin doğruluk oranını ve öğrenme hızını ölçmek için doğruluk ve kayıp değer grafikleri çizilmektedir. Kayıp değer azaldıkça kullanılan modelin doğruluk oranı yani başarısı artmaktadır. Diğer yandan, derin öğrenme modellerinin diğer geleneksel algoritmalar ile birlikte uygulanması sonucu elde edilen performans ölçütleri Tablo 5 ile verilmektedir. Önerilen Inception-ResNet-V2 derin öğrenme modeli ile eğitim aşamasından sonra elde edilen doğruluk ve kayıp değer grafikleri Şekil 10 ile verilmektedir.

\begin{tabular}{|c|c|c|c|c|c|}
\hline & $\mathrm{ACC}$ & FSC & KAPPA & REC & PRE \\
\hline InceptionResnetV2+KNN & $\% 85.80$ & 0.83 & 0.14 & 0.86 & 0.82 \\
\hline InceptionResnetV2+SVM & $\% 87.03$ & 0.81 & 0.0 & 0.87 & 0.76 \\
\hline InceptionResnetV2+RForest & $\% 86.58$ & 0.83 & 0.14 & 0.87 & 0.82 \\
\hline InceptionResnetV2+AdaBoost & $\% 86.92$ & 0.82 & 0.07 & 0.87 & 0.82 \\
\hline InceptionResnetV2+XGB & $\% 8673$ & 0.83 & 0.16 & 0.87 & 0.83 \\
\hline VGG19+KNN & $\% 88.08$ & 0.84 & 0.19 & 0.88 & 0.87 \\
\hline VGG19+RForest & $\% 88.84$ & 0.86 & 0.28 & 0.89 & 0.88 \\
\hline VGG19+AdaBoost & $\% 88.58$ & 0.87 & 0.41 & 0.89 & 0.87 \\
\hline VGG19+XGB & $\% 90.84$ & 0.90 & 0.52 & 0.91 & 0.90 \\
\hline DenseNet169+KNN & $\% 89.43$ & 0.88 & 0.42 & 0.89 & 0.88 \\
\hline DenseNet169+RForest & $\% 90.38$ & 0.89 & 0.46 & 0.90 & 0.89 \\
\hline DenseNet169+AdaBoost & $\% 90.75$ & 0.90 & 0.56 & 0.91 & 0.90 \\
\hline DenseNet169+XGB & $\% 92.45$ & 0.92 & 0,63 & 0.92 & 0.92 \\
\hline
\end{tabular}



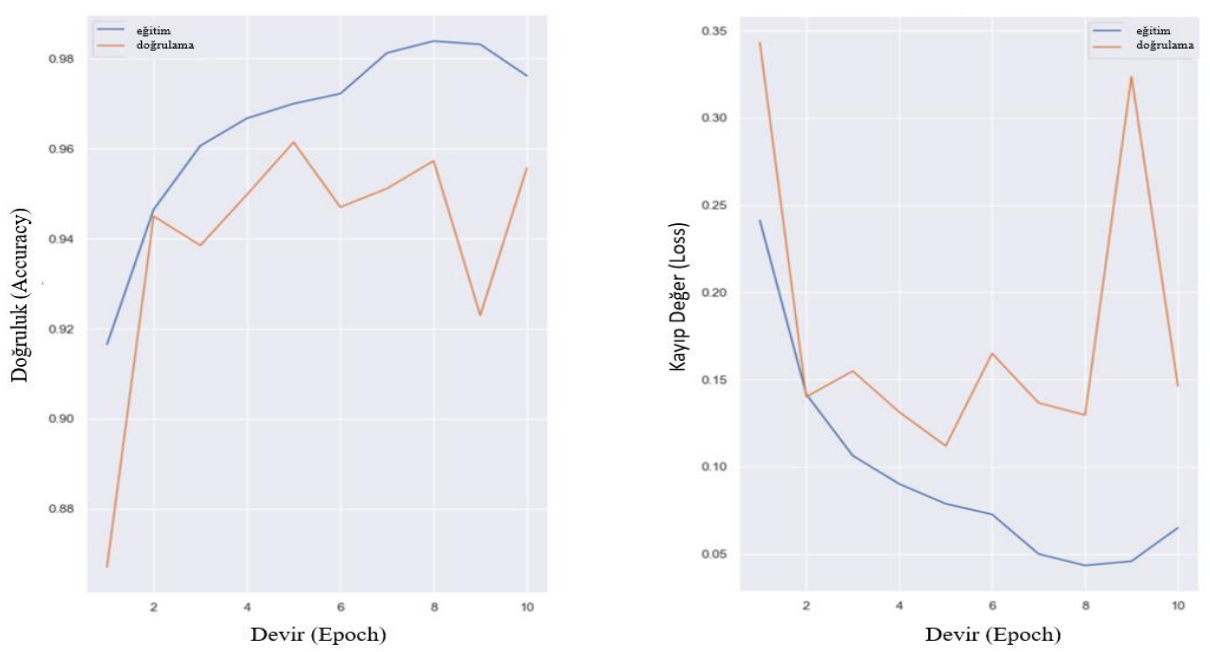

Şekil 10. Önerilen Modelin Doğruluk ve Kayıp Değeri Grafikleri

Tablo 5'de derin öğrenme modelleri, makine öğrenmesi algoritmalarından k-en yakın komşu (KNN), destek vektör makineleri (SVM), rasgele orman (RForest), AdaBoost ve aşırı gradyan artırma (XGBoost) ile birlikte uygulanarak performansları karşılaştırılmıştır. Tablo 4 ve Tablo 5 incelendiğinde önerilen InceptionResnetV2 derin öğrenme modelinin, diğer modellere göre üstünlüğünü kanıtlamaktadır.

\section{SONUÇ}

Meme kanseri, en yaygın görülen ve ölümlere sebebiyet veren kanser türleri arasındadır. Meme kanserinde ölüm oranlarını azaltmanın en iyi yöntemi hastalığın erken teşhisidir. Bu çalışmada, meme kanserinin erken teşhis edilebilmesi için Inception-ResNet-V2 derin öğrenme modeli önerilmiştir. Inception-ResNet-V2 modeli Inception ve ResNet modellerinin bir araya getirilmesi ile oluşturulan melez bir modeldir. Önerilen derin öğrenme modeli ile \%96.21 doğruluk, \%97.48 geri çağırma, \%98.18 kesinlik, \%97.83 F-ölçütü, \%98.00 eğri altında kalan alan ve 0.83 Cohen kappa performans değerleri elde edilmiştir. Meme kanseri tespitinde kötü huylu tümörlerin tespiti oldukça önemlidir. Sonuçlar değerlendirildiğinde, önerilen derin öğrenme mimarisiyle hem iyi huylu hem de kötü huylu tümörlerin en iyi şekilde tahmin edildiği görülmektedir. Gelecekte, önerilen derin öğrenme mimarisinin farklı önişleme adımları ve makine öğrenmesi algoritmaları ile bir araya getirilmesi ile hata oranlarının enaza indirgenmesi hedeflenmektedir. Ayrıca, farklı veri setleri ile önerilen modelin etkinliği ve gürbüzlüğünün kanıtlanması planlanmaktadir.

Gelecekte farklı derin öğrenme mimarileri kullanılarak geniş ve derin bir veri seti üzerinde doğruluk değerini artırmaya yönelik çalışmaların yapılması ve modelin etkinliği ve gürbüzlügüüün kanıtlanması hedeflenmektedir. Derin öğrenme alanındaki donanımsal gelişmeler takip edilerek gögüs kanseri teşhisinde yapılacak farklı çalışmalar ile literatüre önemli katkıların yapılması planlanmaktadır.

\section{KAYNAKLAR}

[1] Anonim [İnternet]. Kanser Nedir?; 2021[Erişim 5 Haziran 2021] Erişim Linki: https://hsgm.saglik.gov.tr/tr/kanser-nedir-belirtileri

[2] Akram M., Iqbal M., Daniyal M., Khan A. U. Awareness and current knowledge of breast cancer. Biological research. 2017;50(1): 1-23.

[3] Bray F., Ferlay J., Soerjomataram I., Siegel R. L., Torre L. A., Jemal A. Global cancer statistics 2018: GLOBOCAN estimates of incidence and mortality worldwide for 36 cancers in 185 countries. Cancer Journal for Clinicians. 2018;68(6):394-424.

[4] Wild C. P., Weiderpass E., Stewart B. W. World Cancer Report: Cancer Research for Cancer Prevention. International Agency for Research on Cancer. Lyon, France, http://publications.iarc.fr/586. Licence: CC BYNC-ND 3.0 IGO, 2020.

[5] Duggento A., Aiello M., Cavaliere C., Cascella G. L., Cascella D., Conte G., Guerrisi M., Toschi N. An ad hoc random initialization deep neural network architecture for discriminating malignant breast cancer lesions in mammographic images. Contrast media \& molecular imaging. 2019.

[6] Gastounioti A., Conant E. F., Kontos D. Beyond breast density: a review on the advancing role of parenchymal texture analysis in breast cancer risk assessment. Breast cancer research. 2016;18(1):112.

[7] Jadoon M. M., Zhang Q., Haq I. U., Butt S., Jadoon A. Three-Class Mammogram Classification Based on Descriptive CNN Features. BioMed research international. 2017.

[8] Hepsă̆ P. U., Özel S. A., Yazıcı A. Using deep learning for mammography classification. International Conference on Computer Science and Engineering (UBMK). 2017;418-423. doi: 10.1109/UBMK.2017.8093429.

[9] Nahid A., Mehrabi M. A., Kong Y. Histopathological breast cancer image classification by deep neural network techniques guided by local clustering. BioMed research international. 2018. 
[10] Zhang Y., Pan C., Chen X., Wang F. Abnormal breast identification by nine-layer convolutional neural network with parametric rectified linear unit and rank-based stochastic pooling. Journal of Computational Science.2018;27:57-68.

[11] Sivasakthiselvan S., Sahoo S., Panda A., Mishra R. Image classification toward breast cancer. International Journal of Research in Engineering and Science. 2018;6(8):129-139.

[12] Behera A., Behera S., Das F., Kumar B. Malignant classification of mammogram images based on deep learning. International Journal of Research in Engineering and Science. 2018;6(9):35-46.

[13] Ahmed A. H., Salem M. A. M. Mammogram Based Cancer Detection Using Deep Convolutional Neural Networks. 13th International Conference on Computer Engineering and Systems (ICCES). 2018;694-699.

[14] Ragab D. A., Sharkas M., Marshall S., Ren J. Breast cancer detection using deep convolutional neural networks and support vector machines. PeerJ. 2019.

[15] Li H., Zhuang S., Li D., Zhao J., Ma Y. Benign and malignant classification of mammogram images based on deep learning. Biomedical Signal Processing and Control. 2019;51:347-354.

[16] Chanda P. B., Sarkar S. K. Detection and Classification of Breast Cancer in Mammographic Images Using Efficient Image Segmentation Technique. Advances in Control. Signal Processing and Energy System. 2020;107-117.

[17] Trivizakis E., Ioannidis G. S., Melissianos V. D., Papadakis G. Z., Tsatsakis A., Spandidos D. A., Marias K. A novel deep learning architecture outperforming 'off-the-shelf' transfer learning and feature-based methods in the automated assessment of mammographic breast density. Oncology reports. 2019;42(5):2009-2015.

[18] Yu X., Pang W., Xu Q., Liang M.. Mammographic image classification with deep fusion learning. Scientific Reports. 2020;10(1):1-11.

[19] He K., Zhang X., Ren S., Sun J. Deep residual learning for image recognition. IEEE conference on computer vision and pattern recognition. 2016. 770778. 